\title{
Simple Models for Diffusion in Thin Plates or Membranes
}

\author{
Jorge Corrêa de Araújo ${ }^{(0)}$, Rosa García Márquez ${ }^{\circledR}$ \\ State University of Rio de Janeiro, DMAT-FFP, Rio de Janeiro, Brazil \\ Email: jcaraujo_55@yahoo.com.br, rosagmarquez@yahoo.com.br
}

How to cite this paper: de Araújo, J.C. and Márquez, R.G. (2019) Simple Models for Diffusion in Thin Plates or Membranes. Journal of Applied Mathematics and Physics, 7, 1547-1559.

https://doi.org/10.4236/jamp.2019.77105

Received: June 26, 2019

Accepted: July 21, 2019

Published: July 24, 2019

Copyright $\odot 2019$ by author(s) and Scientific Research Publishing Inc. This work is licensed under the Creative Commons Attribution International License (CC BY 4.0).

http://creativecommons.org/licenses/by/4.0/

c) (i) Open Access

\begin{abstract}
Two simplified models, linear and nonlinear, were used in a cementation process on a homogeneous thin carbon steel plate. The parameters for these models, as obtained by the least squares' method the first one in a global way while the other parameters refer to the second model-were estimated by a set of local minimums. To compare the performance of these models we used theoretical data, for the same diffusion problem obtained by a one-dimensional transient model considering the concentrations in the mean plane of the plate. The results for carbon concentrations in weight percentage in the plate $(\% \mathrm{pC})$ as a time-only dependent function with these simplified models to represent the analyzed diffusion process were in good agreement with those from a stricter model. The diffusion flows of these models were determined and a reasonable agreement can be seen in relation to the flow obtained by the theoretical model on the surface of the plate. This study shows that it is possible to use this methodology with the given restrictions adopted here to describe the concentration and the diffusion flow of other solutes in thin membranes.
\end{abstract}

\section{Keywords}

Simplified Model, Theoretical Model, Membrane, Mean Concentration

\section{Introduction}

The one-dimensional diffusion in a medium is limited by two parallel planes, for example, in $x=0$ and $x=L$ of such thin thickness, so that the entire diffusive process occurs through these sheets or membranes, while only a negligible amount occurs through the lateral faces, which is well known [1] [2]. In particular, if on the face $x=0$ the concentration of the diffusing substance is constant and equal to $C_{1}$ and on the other $x=L$ it is a constant $C_{2}$, and if the initial 
concentration is uniform and equal to a constant $C_{0}$, the concentration $C(x, t)$ can be obtained from the transient diffusion equation in a one-dimensional medium with a constant diffusion coefficient $D$ given by $D \frac{\partial C^{2}}{\partial x^{2}}=\frac{\partial C}{\partial t}$ [2] using, for example, the method of separating variables or through the Laplace Transform [3]. The solution of this problem via the separation of variables involves an infinite series of sine terms combined with an exponential function with a negative factor in each term of the series, which albeit complicated, quickly converges, except for small values of [4]. The solution obtained by the Laplace Transform lies in the calculation of the $\operatorname{erf}(x)$ function or Gauss error function, whose values are given in tables for different values of $\frac{x}{2 \sqrt{D t}}$. In general, diffusion is three-dimensional process, but sometimes the problem is simplified using a smaller number of dimensions. According to Fox et al. [5], for many problems found in engineering, a one-dimensional analysis is adequate to provide approximate solutions with the precision required in engineering practice. Recently, Araújo e Márquez [6] used transient one-dimensional diffusion in a cementing process of a homogeneous metal sample of carbon steel with a thickness of less than two millimeters to obtain information on carbon concentration in a semi-infinite plate where the Gauss error function was replaced by a fifth-degree polynomial.

The cementation process consists of the hardening of the surface of steel to higher levels to that of its interior by the diffusion or transport of carbon atoms at high temperatures in an atmosphere rich in hydrocarbon gas such as $\mathrm{CH}_{4}$ methane gas [7]. The question we pose in this article is whether there is a simpler model that can be adopted to estimate the concentration of carbon in diffusion in thin plates or membranes. Although recognizing that the process of diffusion of materials through cell membranes is quite complicated, Bassanezi and Ferreira Jr. [8] and Bassanezi [9] presented two diffusion models through cell membranes based on a simplification of Fick's Law [7]. These diffusion models are applied when the concentration difference between the cell medium and the homogeneous liquid medium where the cell is immersed is small and the cell has the area and volume constant throughout the process. For this, it is also assumed in a natural way that the flow of molecules goes in both directions until the concentration inside the cell is equal to the concentration of the medium it is suspended in. The first model is represented by the linear ordinary differential equation given by

$$
\frac{\mathrm{d} C}{\mathrm{~d} t}=\frac{k A}{V}\left[C_{e}-C(t)\right],
$$

where $C_{e}$ is the concentration of the medium surrounding the membrane, and $k$ is a membrane permeability constant, $A$ is the surface area, $V$ is the constant volume of the cell, while $C(t)$ it is the concentration of the diffusing solute in the cell in $t$ time. The $k$ constant depends on each solution, the thickness and the 
structure of the membrane, so it needs to be estimated for each situation. The second model, also simplified, but with two parameters, is given by an ordinary non-linear differential equation as

$$
\frac{\mathrm{d}\left[C(t)-C_{e}\right]}{\mathrm{d} t}=\frac{A}{V}\left[k_{1}\left(C_{e}-C(t)\right)+k_{2}\left(C_{e}-C(t)\right)^{2}\right] .
$$

The term in square brackets is the function for solute flowing into the cell [8]. According to Bassanezi and Ferreira Jr. [8], the experimental obtaining of the constant can be difficult and sometimes impossible to obtain, in which case the two-parameter model can be useful for the theoretical analysis of the problem understudy, but not for the specific study. Although agreeing that the experimental achievement of these parameters is not trivial, their estimates are possible in our understanding, provided that experimental data $\left(t_{i}, C\left(t_{i}\right)\right)$ can be obtained by some experimental or theoretical means. In this sense, the purpose of this paper is to solve in detail the differential equation of the model with two parameters and, from the theoretical data estimated by a transient one-dimensional diffusion model of carbon diffusion in a thin homogeneous sample of carbon steel of thickness to one millimeter, to estimate the $k_{1}$ parameter of the linear model using the linear least squares' or linear regression method and the $k_{1}$ and $k_{2}$ parameters of the non-linear model, using the non-linear least squares' method. These parameters are related to the medium where the diffusion occurs.

The results show that there was good agreement among the models adopted in this study with the results of the carbon concentrations estimated by the one-dimensional model of transient diffusion on the plane of the plate $x=0.0005 \mathrm{~m}$ or membrane. Thus, the simplified models, in particular the one-parameter model, gave a good description of the problem analyzed, providing a semi-qualitative mean value for the concentration within the plate at any point and instant in the $x$ direction.

\section{Materials and Methods}

Consider a homogeneous metal plate formed by an Iron-gamma Carbon alloy, indicated by, $\mathrm{Fe}_{\gamma}-\mathrm{C}$ which hast to be hardened through a process of cementation [7]. The steel part is exposed in an atmosphere rich in hydrocarbon gas (such as methane gas, $\mathrm{CH}_{4}$ ), under $\mathrm{T}=1000^{\circ} \mathrm{C}$. The homogeneous plate with the properties shown in Table 1, has an uniform concentration of carbon $C_{0}=C(x, 0)=0.08 \% \mathrm{pC}$ (weight percent carbon) for $0<x<L$. The carbon concentration is kept constant and equal $C_{1}=C(0, t)=0.10 \% \mathrm{pC}$ to the flat face $x=0$, and also constant and equal $C_{2}=C(L, t)=0.10 \% \mathrm{pC}$ to the flat face $x=L=0.001 \mathrm{~m}$ with $t \geq 0$. Under these conditions, the goal is to obtain theoretical data on the process at different moments in time, in hours for the mean plane of the plate, that is, in $x=0.0005 \mathrm{~m}$. Figure 1 shows in simplified form the metal plate subjected to the conditions given in the cementation process described above.

The thickness is so small that it will be assumed that the entire diffusion process happens through these sheets or membranes, while a negligible amount 


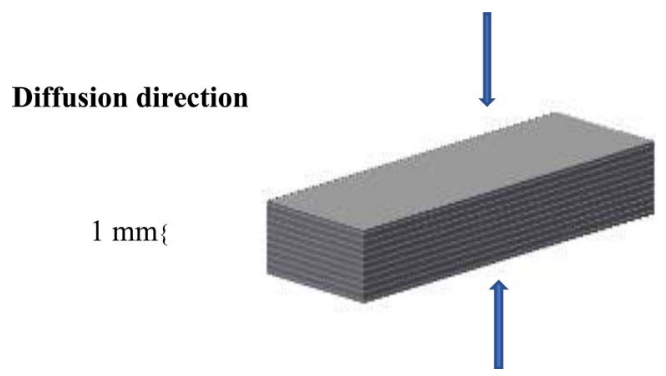

Figure 1. Carbon transient diffusion moving through a thin carbon steel plate.

Table 1. Parameters considered in the cementation process.

\begin{tabular}{ccc}
\hline PROCESS PARAMETERS & NOTATION & VALUES \\
\hline Surface area of the plate & $A$ & $29.4 \times 10^{-4} \mathrm{~m}^{2}$ \\
Volume of the plate & $V$ & $29.5 \times 10^{-7} \mathrm{~m}^{3}$ \\
Board thickness & $x_{L}$ & $L=0.001 \mathrm{~m}$ \\
Diffusion Rate & $D_{o}$ & $2.3 \times 10^{-5} \mathrm{~m}^{2} / \mathrm{s}^{*}$ \\
C initial concentration & $C(x, 0)=C_{o}, 0<x<L$ & $0.08 \% \mathrm{pC}$ \\
C concentration on faces & $C(0, t)=C_{1} ; C(L, t)=C_{2}$ & $0.10 \% \mathrm{pC}$ \\
Diffusion temperature & $T$ & $1000^{\circ} \mathrm{C}^{*}$ \\
Activation energy & $Q d$ & $148 \mathrm{~kJ} / \mathrm{mol}^{*}$ \\
Constant of the gas & $R$ & $8.31 \mathrm{~J} / \mathrm{mol}^{*} \cdot \mathrm{K}^{*}$ \\
Iron density range & $\rho_{\mathrm{Fe}_{y}}$ & $7.86 \mathrm{~g} / \mathrm{cm}^{3 * *}$ \\
Carbon density & $\rho_{\mathrm{C}}$ & $2.267 \mathrm{~g} / \mathrm{cm}^{3 * * *}$
\end{tabular}

${ }^{*}$ Reference value obtained for this diffusion (Callister, 2002, p. 70, Table 5.2). ${ }^{*}$ Reference value obtained for this diffusion (Callister, 2002, p. 537, Table B.1). ${ }^{* \star}$ https://iupac.org/. Union of Pure and Applied Chemistry (IUPAC)

occurs through the lateral faces [1] [2].

Table 1 shows the parameters for the carburizing process, notation and assumed values, and the initial conditions of the diffusion process.

\subsection{Linear Least Squares: Discrete Case}

Given a set of points $\left(x_{i}, y_{i}=f\left(x_{i}\right)\right), i=1, \cdots, n$ and $a \leq x_{i} \leq b$, the goal is to choose $g_{i}(x)$ continuous real functions in the $I=[a, b]$ interval to obtain constants or parameters $\lambda_{1}, \cdots, \lambda_{n}$ so that

$$
\phi(x)=\sum_{i=1}^{n} \lambda_{i} g_{i}(x) \cong f(x) .
$$

As the $\lambda_{i}$ coefficients appear linearly in the definition of the $\varphi(x)$ approximation function, this model is called linear [10]. The choice of $\varphi(x)$ model depends on the $\left(x_{i}, y_{i}=f\left(x_{i}\right)\right)$ dispersion diagram, or in other words of the tabulated points. Be $R_{k}=f\left(x_{k}\right)-\phi\left(x_{k}\right)$ the $k$-th residue of this approach. The objective is to minimize linear functional 


$$
F(\lambda)=F\left(\lambda_{1}, \cdots, \lambda_{n}\right)=\sum_{i=1}^{m} R_{i}^{2}
$$

where $m \gg n$. To get a $F(\lambda)$ minimum point of it is necessary to solve the equation of the critical points given by

$$
\nabla F(\lambda)=\overrightarrow{0}
$$

The equations obtained from Equation (5) give rise to a linear system of $n \times n$ order given by

$$
A \lambda=b,
$$

where, according to Ruggiero e Lopes [10] matrix entries can be obtained by $a_{i j}=\left\langle\bar{g}_{i}, \bar{g}_{j}\right\rangle ; b_{i}=\left\langle\bar{y}, \bar{g}_{i}\right\rangle, \quad \bar{g}_{i}=\left(g_{i}\left(x_{1}\right), \cdots, g_{i}\left(x_{m}\right)\right)^{\mathrm{T}}$ being $\bar{y}=\left(f\left(x_{1}\right), \cdots, f\left(x_{m}\right)\right)^{\mathrm{T}}$, with $i, j=1, \cdots, n$ and $\langle$,$\rangle denotes the scalar product$ in $I R^{m}$ space. If the matrix is invertible, it is obtained $\lambda=\left(\lambda_{1}, \cdots, \lambda_{n}\right)$ in an unique way of Equation (6).

\subsection{Nonlinear Least Squares: Discrete Case}

If $\phi(x)$ it is not a linear model of the parameters as in Equation (3), the equation of the critical points no longer produces a linear system as obtained in Equation (6). Consider

$$
\begin{aligned}
& R: U \subset I R^{n} \rightarrow I R^{m} \\
& R(\lambda)=\left(R_{1}(\lambda), \cdots, R_{m}(\lambda)\right)^{\mathrm{T}} .
\end{aligned}
$$

Therefore, as $R(\lambda)$ is a vector function for residues in the $R^{m}$ space and $\varphi_{i}(\lambda)=\varphi\left(x_{i}, \lambda\right)$ is the nonlinear model for adjustment point $\left(x_{i}, y_{i}=f\left(x_{i}\right)\right)$, $i=1, \cdots, m$ and $a \leq x_{i} \leq b$, where $\lambda=\left(\lambda_{1}, \cdots, \lambda_{n}\right)^{\mathrm{T}}$ is a vector of adjustable parameters of the $I R^{n}$ space. With this notation, the $k$-th residue of this approximation is defined by $R_{k}(\lambda)=f\left(x_{k}\right)-\phi\left(x_{k}, \lambda\right), k=1, \cdots, m$. The objective is to minimize $F(\lambda)$ as in Equation (4), that is, the Equation (5) of the critical points is written thus

$$
\sum_{i=1}^{m} R_{i}(\lambda) \frac{\partial \phi\left(x_{i}, \lambda\right)}{\partial \lambda_{k}}=0, \quad k=1, \cdots, n
$$

Using a Taylor series expansion [11] to the first order for each $R_{i}(\lambda)$ coordinate function around $\lambda$, being $p=\left(p_{1}, \cdots, p_{n}\right)^{\mathrm{T}}$ an increment vector we have

$$
R_{i}(\lambda+p)=R_{i}(\lambda)+\nabla R_{i}(\lambda) p,
$$

where $\nabla R_{i}(\lambda)=\left(\frac{\partial R_{i}(\lambda)}{\partial \lambda_{1}}, \cdots, \frac{\partial R_{i}(\lambda)}{\partial \lambda_{n}}\right)$, with $i=1, \cdots, m$.

From Equations $((8),(9))$ it is $k=1, \cdots, n$

$$
\sum_{i=1}^{m}\left[R_{i}(\lambda)+\sum_{j=1}^{n} \frac{\partial \phi\left(x_{i}, \lambda\right)}{\partial \lambda_{j}} p_{j}\right] \frac{\partial \phi\left(x_{i}, \lambda\right)}{\partial \lambda_{k}}=0 .
$$

The Jacobian matrix of the $R(\lambda)$ transformation is an $m \times n$ order matrix 
given by

$$
J(\lambda)=\left[\begin{array}{ccc}
\frac{\partial \phi\left(x_{1}, \lambda\right)}{\partial \lambda_{1}} & \cdots & \frac{\partial \phi\left(x_{1}, \lambda\right)}{\partial \lambda_{n}} \\
\vdots & \ddots & \vdots \\
\frac{\partial \phi\left(x_{m}, \lambda\right)}{\partial \lambda_{1}} & \cdots & \frac{\partial \phi\left(x_{m}, \lambda\right)}{\partial \lambda_{n}}
\end{array}\right] .
$$

From Equations ((10), (11)) we have a linear system of $n \times n$ order given by

$$
J^{\mathrm{T}}(\lambda) J(\lambda) p=-J^{\mathrm{T}}(\lambda) R(\lambda) .
$$

Equation (12) is the basis for an iterative process and is known as a modified Newton method [12]. Taking $p^{0}=\left(p_{1}^{0}, \cdots, p_{n}^{0}\right)^{\mathrm{T}}$ as an initial approximation, we calculate $J(\lambda), J^{\mathrm{T}}(\lambda)$ and $R(\lambda)$ using $p^{0}$. Then the system given by Equation (10) is solved to obtain the $p$ value. The vector $p^{1}=p^{0}+p$ is evaluated, for example, by the sum of the quadratic residuals, that is, if $\left\|R\left(p^{1}\right)\right\|^{2}=\sum_{i=1}^{m} R_{i}\left(p^{1}\right)^{2}<$ tol then, $p^{1}$ it is a solution by least squares, where tol is the tolerance allowed in the approximation. If $p^{1}$ it is not the best solution for the least squares, new iterations are performed until convergence can be achieved.

\subsection{The One-Dimensional Diffusion Equation}

The solution of the transient one-dimensional diffusion equation for the diffusion problem in thin membranes with constant surface concentrations and initial distribution with uniform concentration, that is,

$$
C_{1}=C(0, t), C_{2}=C(L, t) ; t \geq 0 \text { and } C_{0}=C(x, 0) ; 0<x<L,
$$

can be obtained by the method of separation of variables, whose solution is given according to Crank [2] by

$$
\begin{aligned}
C=C(x, t)= & C_{1}+\left(\frac{C_{2}-C_{1}}{L}\right) x+\frac{2}{\pi} \sum_{n=1}^{\infty}\left(\frac{C_{2} \cos (n \pi)-C_{1}}{n}\right) \sin \left(\frac{n \pi x}{L}\right) \mathrm{e}^{\frac{-D n^{2} \pi^{2} t}{L^{2}}} \\
& +\frac{4 C_{0}}{\pi} \sum_{m=0}^{\infty}\left(\frac{1}{2 m+1}\right) \sin \left(\frac{(2 m+1) \pi x}{L}\right) \mathrm{e}^{\frac{-D(2 m+1)^{2} \pi^{2} t}{L^{2}}}
\end{aligned}
$$

\section{Results and Discussions}

Model 1 as given and reported by Equation (1) is given by

$$
\frac{\mathrm{d} C}{\mathrm{~d} t}=\frac{k A}{V}\left[C_{e}-C(t)\right],
$$

as proposed by Bassanezi [9] and based on Fick's Law for the diffusion of materials through permeable membranes [4]. In Equation (15), the permeability constant of the membrane can be given, for example, in $\mathrm{m} / \mathrm{s}$ and where $C(t)$ represents the diffusion solute concentration inside the cell. Equation (15) can be solved by separating variables, that is, 


$$
\int_{C_{0}}^{C} \frac{\mathrm{d} C}{C_{e}-C(t)}=k \frac{A}{V} \int_{0}^{t} \mathrm{~d} t
$$

From Equation (16) results the $C(t)$ expression for given by

$$
C=C(t)=\left(C_{0}-C_{e}\right) \mathrm{e}^{-k \frac{A}{V} t}+C_{e},
$$

where $C_{0}=C(0)$. Note that $C(t) \rightarrow C_{e}$ when $t \rightarrow \infty$.

In Equation (15), an analogy with Fick's first law for one-dimensional diffusion in a steady state [7], making it possible to interpret the term $J=k \cdot\left[C_{e}-C(t)\right]$ as the amount of mass that crosses the membrane per unit area, in a given direction per unit of time. Model 2, as proposed by Bassanezi and Ferreira [8] and already mentioned in the introduction (Equation (2)) is given formally by

$$
\frac{\mathrm{d}\left[C(t)-C_{e}\right]}{\mathrm{d} t}=\frac{A}{V}\left[k_{1}\left(C_{e}-C(t)\right)+k_{2}\left(C_{e}-C(t)\right)^{2}\right] .
$$

Making $f(C)=F\left(C_{e}-C\right)$ where $F\left(C_{e}-C\right)$ it is denominated by the authors as a function for flow based on the difference of concentrations, where $f(C)$ is a real function of a real variable, and where $f\left(C_{e}\right)=F\left(C_{e}-C_{e}\right)=F(0)=0$.

To justify this fact we assume the plausibility where if there is no difference of concentration there should be no flow between the means. Assuming $f(C)$ is at least $C^{3}(I, R)$ class function; where $I=\left[0, t_{0}\right]$ [11] using a Taylor series expansion of up to the second order around has [11],

$$
f\left(C_{e}-h\right)=f\left(C_{e}\right)+f^{\prime}\left(C_{e}\right)\left(C_{e}-C(t)\right)+\frac{f^{\prime \prime}\left(C_{e}\right)}{2}\left(C_{e}-C(t)\right)^{2},
$$

or,

$$
f(C)=F^{\prime}(0)\left(C_{e}-C(t)\right)+\frac{F^{\prime \prime}(0)}{2}\left(C_{e}-C(t)\right)^{2} .
$$

From Equation (15) we have seen that $J=k \cdot\left[C_{e}-C(t)\right]$ represents a flow of molecules into the cell, then replacing that term with the given $f(C)$ flow function as in Equation (18), we obtain the two-parameter formulation for cell diffusion only reported by Bassanezi and Ferreira Jr. [8] to obtain Equations (2) or (18),

$$
\frac{\mathrm{d}}{\mathrm{d} t}\left(C-C_{e}\right)=\frac{A}{V}\left[k_{1}\left(C_{e}-C(t)\right)+k_{2}\left(C_{e}-C(t)\right)^{2}\right],
$$

where, $k_{1}=k F^{\prime}(0)$ and $k_{2}=k \frac{F^{\prime \prime}(0)}{2}$. For the physical sense of the problem, the unity of $k_{1}$ is the same as of $k$, that is, $\mathrm{m} / \mathrm{s}$, while the unity $k_{2}$ of can be given as, $\mathrm{m}^{4} / \mathrm{kg} \cdot$ s. Making $y=C-C_{e}$ the Equation (21) rewrite in the form

$$
\frac{\mathrm{d} y}{\mathrm{~d} t}+\frac{k_{1} A}{V} y=\frac{k_{2} A}{V} y^{2} .
$$

Equation (22) is a Bernoulli equation [13] and can be solved by changing the variable as $u=y^{-1}$, to have Equation (22) then in the linear form given by 


$$
\frac{\mathrm{d} u}{\mathrm{~d} t}-\frac{k_{1} A}{V} u=-\frac{k_{2} A}{V} .
$$

Equation (23) can be solved using the $\mu(t)=\mathrm{e}^{-\frac{k_{1} A}{V} t}$ integral factor to obtain

$$
u=\frac{K k_{1} \mathrm{e}^{\frac{k_{1} A}{V} t}+k_{2}}{k_{1}} \text {. }
$$

Using the fact that $u=y^{-1}$ and $y(0)=C_{0}-C_{e}$ follows from Equation (23) the solution to Equation (21) given by

$$
C=C_{e}+\frac{k_{1}\left(C_{0}-C_{e}\right)}{k_{2}\left(C_{0}-C_{e}\right)+\mathrm{e}^{\frac{k_{1} A}{V} t}\left[k_{1}-k_{2}\left(C_{0}-C_{e}\right)\right]} .
$$

Note what $C(0)=C_{0}$ and $C(t) \rightarrow C_{e}$ when $t \rightarrow \infty$. In particular, if $F^{\prime}(0)=1$ and $F^{\prime \prime}(0)=0$ and only then, the differential equation describing model 1, as given by Equation (13) is retrieved. However, if $F^{\prime}(0) \neq 1$ and $F^{\prime \prime}(0)=0$, the constant $k_{1}=k F^{\prime}(0) \neq k$, and in this sense, Model 2 is not exactly an extension of Model 1, but the analytical form of Model 2 is an extension of Model 1. To estimate the parameters of Equations (17) and (24) we will use the theoretical data obtained from the cementation process, whose parameters are shown in Table 1 obtained by means of Equation (12), that is, the one-dimensional transient diffusion model (MD) or as we will call theoretical model.

Table 2 shows the estimates in \%pC at the $x=0.0005 \mathrm{~m}$ plane position in several time using ten terms of the series defined by the Equation (14), so that the values with number of major terms would not provide significant concentrations variations. The choice of the mean plane of the plate or membrane was to assume that the solute concentration at this depth of the plate would represent a mean concentration (inside the plate), and thus, the estimates obtained for the parameters of the simplified models would "mirroring" in a semi-qualitatively way this mean concentration.

The permeability parameter (Model 1) can be obtained through the global minimum with the linearization of Equation (15) according to the procedure

Table 2. Estimates for \%pC in the membrane with MD.

\begin{tabular}{cc}
\hline Time (hours) & \% $(\mathrm{MD})$ \\
\hline 0.0 & 0.0008000 \\
0.5 & 0.0008231 \\
1.5 & 0.0009089 \\
2.0 & 0.0009353 \\
3.0 & 0.0009674 \\
5.0 & 0.0009917 \\
7.0 & 0.0009979 \\
9.0 & 0.0009990 \\
\hline
\end{tabular}


described in Section 2.1. doing $y=C-C_{e}$, and therefore Equation (15) is rewritten thus

$$
-y=\left(C_{e}-C_{0}\right) \mathrm{e}^{\frac{A k}{V} t} .
$$

In applying the Neperian Logarithm to Equation (26) we obtain

$$
z=\ln (-y)=\ln \left(C_{e}-C_{0}\right)-\frac{k A}{V} t .
$$

Thus, using the discrete points in Table 2 we can write

$$
z_{i}=B+\lambda t_{i},
$$

where $z_{i}=\ln \left(C_{e}-C\left(t_{i}\right)\right), B=\ln \left(C_{e}-C_{0}\right)$ and $\lambda=-\frac{k A}{V}$. As $B$ is constant, Equation (28) can be reduced to a linear form given by

$$
w_{i}=\lambda t_{i},
$$

which $w_{i}=z_{i}-B$. Therefore Equation (28) can be solved by rewriting Equation (1) as, $\varphi(t, \lambda)=\lambda t$ being $g_{1}(t)=t$. Thus, the matrix of the system given by Equation (4) is of an order of $1 \times 1$, that is, $a_{11}=\left\langle\bar{g}_{1}, \bar{g}_{1}\right\rangle=\sum_{i=1}^{7} t_{i}^{2}$, $b_{1}=\left\langle\bar{y}, \bar{g}_{1}\right\rangle=\sum_{i=1}^{7} t_{i} w_{i}$. Hence, Equation (6) gives the first-order linear equation given by

$$
a_{11} \lambda=b_{1} \Rightarrow \lambda=-\frac{104.4298}{170.50}=-0.612491 .
$$

As a result, $\lambda=-\frac{k A}{V}$ and $A, V$ are given as shown in Table 1,

$k=6.12491 \times 10^{-4}$, with $\left\|R\left(p^{0}=k\right)\right\|^{2}=\sum_{i=1}^{m} R_{i}\left(p^{0}=k\right)^{2}=1.0441 \times 10^{-9}$. Now Equation (15) is fully determined.

To estimate the Model 2 parameters, we used the non-linear least squares' method described in Section 2.2. From Equation (24) two models were proposed: the model named MD21, where only the $k_{2}$ parameter was allowed to vary while $k_{1}=k$ and Model MD22, where both parameters varied. The initial parameter vector for MD21 was $p_{21}^{0}=0.01$ with a tolerance $t o l=10^{-4}$ and convergence occurring in only two cycles for the value of $k_{2}^{2}=1.9224$, where $\left\|R\left(p_{21}^{2}\right)\right\|^{2}=\sum_{i=1}^{m} R_{i}\left(p_{21}^{2}\right)^{2}=1.0567 \times 10^{-9}$. For MD22, the initial parameter vector was taken as $p_{22}^{0}=(0.0007,1.0)^{\mathrm{T}}$ with a tolerance tol $=10^{-5}$ and convergence also occurring in two cycles for the $p_{22}^{2}=\left(k_{1}^{2}=0.0004, k_{2}^{2}=3.4907\right)^{\mathrm{T}}$, the vector being $\left\|R\left(p_{22}^{2}\right)\right\|^{2}=\sum_{i=1}^{m} R_{i}\left(p_{22}^{2}\right)^{2}=1.0544 \times 10^{-9}$. Please note that in terms of quadratic residues, there is no significant difference between these iterative models.

The relative percentage error for $\mathrm{MD} 1, \varepsilon_{\mathrm{MD} 1}$ in relation to the estimates of concentration in \%pC estimated by the theoretical model MD, is below $3.7 \%$. 
Similarly, for MD21, $\varepsilon_{\mathrm{MD} 21}<6.2 \%$ while for the MD22 model, $\varepsilon_{\mathrm{MD} 22}<6.5 \%$. The highest divergence between concentrations took place in the $t \leq 2.0 \mathrm{~h}$ time interval. Based on the criteria analyzed, and because the MD1 model has its parameter estimated in a global way, and due to its greater simplicity, it is at first the model to be adopted to estimate the mean carbon concentration in the thin plate. Figure 2 shows the adjustment in the half-plane estimated in \%pC obtained with the different adjustment models analyzed.

Figure 2 confirms that the best fit was with MD1 followed by MD22 and finally by MD21 in relation to the theoretical data estimated by the MD model as given by the solid line. The MD22 model when varying the initialization vector $p_{22}^{0}=(0.0007,1.0)^{\mathrm{T}}$ showed instability, probably due to the sensitivity of these parameters and, therefore, the $p_{22}^{2}=\left(k_{1}^{2}=0.0004, k_{2}^{2}=3.4907\right)^{\mathrm{T}}$ was chosen from a set of global minimums. This instability also occurred during the refinement of the parameter in the MD21 model.

One of the advantages of these simplified models is that their expressions for $C(t)$ are analytical, as opposed to the solution obtained by Equation (14) which is given in terms of an infinite series. Figure 2 shows a little more, that is, on the more superficial layers it is expected that models MD21 and MD22 are closer to the estimates of theoretical concentrations, as a greater spread is seen above the continuous graph obtained by the MD model for the mean plate plane.

If we consider the $x=0.00025 \mathrm{~m}$ plane of the plate, the maximum percentage

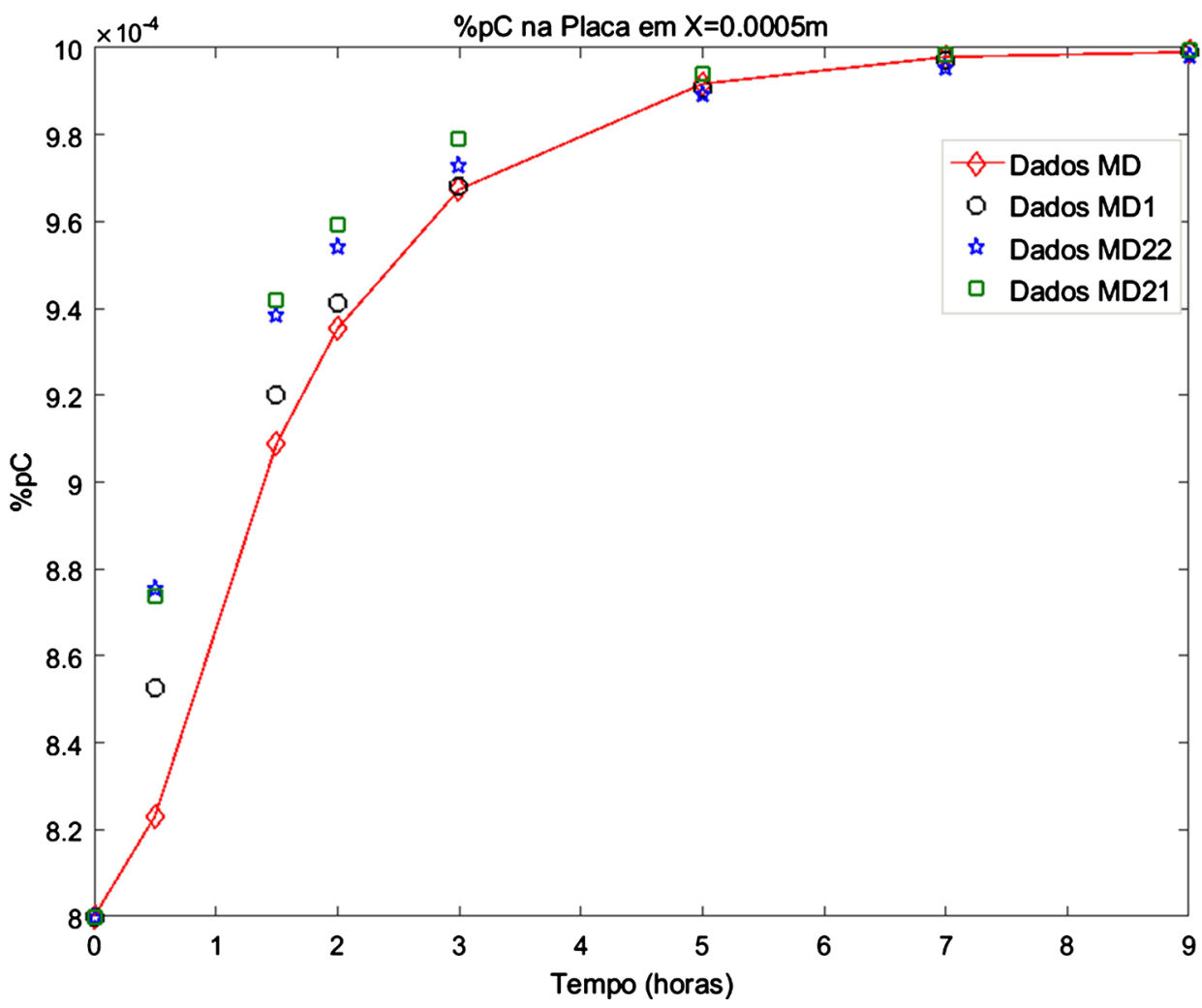

Figure 2. Comparison of the MD1, MD21 and MD22 models with the MD model at the $x=0.0005 \mathrm{~m}$ position. 
difference between the concentrations estimated by the MD1 model for the $x=0.0005 \mathrm{~m}$ plate plane are lower than $2 \%$ when compared with the theoretical data (not shown here), for the estimates of the concentrations in $x=0.00025 \mathrm{~m}$ obtained by the MD model. This allows us to assume that the simplified model to a parameter can estimate, in a semi qualitative way, the $\% \mathrm{pC}$ concentrations inside the plate or thin membrane, and without the need to use iterative methods where the global minimum cannot be obtained. Unlike the MD model, the diffusion of the simplified models lies in the analytical functions. That is, from Equation (15) the diffusion flow function is given by $J_{1}=k \cdot\left[C_{e}-C(t)\right]$, while in Equation (20), the diffusion flow is given by $J_{2}=k_{1}\left(C_{e}-C(t)\right)+k_{2}\left(C_{e}-C(t)\right)^{2}$. From Equation (12) the diffusion flow across the face is given using [2].

$$
\begin{aligned}
J & =-D \frac{\partial C(0, t)}{\partial t} \\
& =-D\left\{\frac{2}{L} \sum_{m=1}^{\infty}\left[C_{2} \cos (m \pi)-C_{1}\right] \mathrm{e}^{-\frac{D m^{2} \pi^{2}}{L^{2}} t}+\frac{4 C_{0}}{L} \sum_{n=0}^{\infty} \mathrm{e}^{-\frac{D(2 n+1)^{2} \pi^{2}}{L^{2}} t}\right\}
\end{aligned}
$$

The flow functions from simple models are dependent on the difference in concentration between the media, so it is to be expected that the $J_{2}$ flow will be less pronounced than the $J_{1}$ flow. Figure 3 clearly shows that the largest difference in concentration occurs in models MD21 and MD22 in relation to the theoretical concentrations, and thus the $C_{e}-C(t)$ terms are smaller in relation

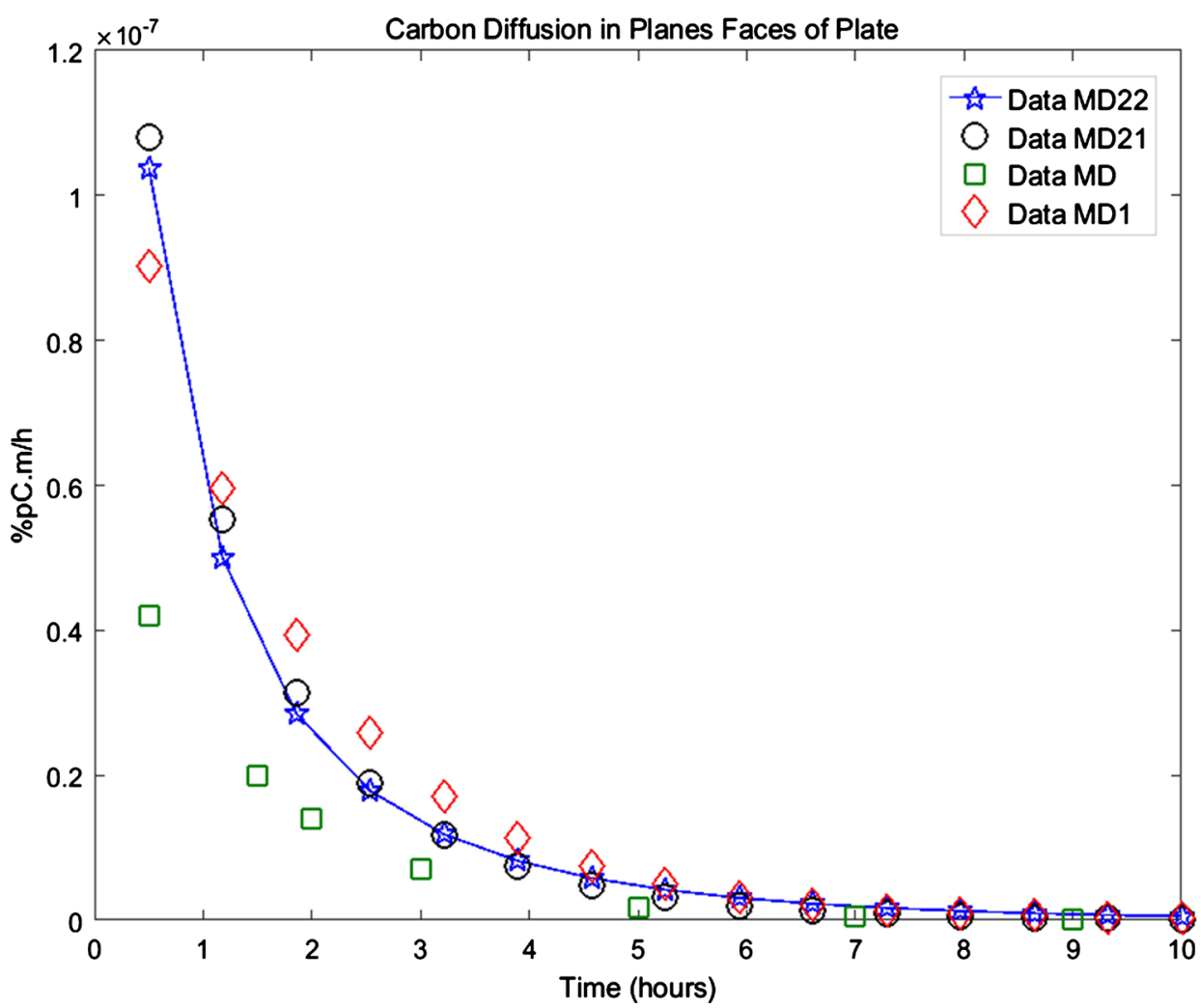

Figure 3. Carbon diffusion estimated for models MD1, MD21, MD22 and MD on the flat faces of the plate. 
to the same terms of the MD1 model, indicating that the diffusion for models MD21 and MD22 is "less apparent" in relation to the $J_{1}$ model. Figure 3 shows the carbon diffusion profile diffusion on the flat faces of the plate, confirming this observation.

After two hours of the cementation process, the carbon transfer rates through the flat section on the surface of the plate were in good agreement when compared with the data provided by the theoretical model. A possible explanation for the discrepancy of flows estimated by the simplified models for times under two hours can be credited to the fact that the parameters estimated for the models were based on the data of the theoretical concentrations obtained for the mean plane, and not the surface plane where the concentration was kept fixed during the cementation process.

\section{Conclusion}

The analyzes showed that the simplified diffusion models in cell membranes analyzed in this study may be an alternative to the transient one-dimensional models used for the description of membrane diffusion processes. The simplest models depend on parameters that can be obtained globally using known function adjustment methods, such as that of the least squares. In particular, they were used to obtain percentages by weight of carbon in a cementation process with restricted thickness conditions. The results obtained were in good agreement when compared with the estimates of the theoretical model used for this purpose. The simplified model with one parameter was shown to be the best option to represent the average estimate of the concentration of carbon solute in the diffusion process due to the concentration difference on the plate or membrane, and this is due to the fact that this model uses only one parameter and that it can be obtained non-iteratively; that is, in a global way.

\section{Conflicts of Interest}

The authors declare no conflicts of interest regarding the publication of this paper.

\section{References}

[1] Carslaw, H.S. and Jaeger, J.C. (2011) Conduction of Heat in Solids. 2nd Edition, Clarendon Press, Oxford.

[2] Crank, J. (2011) The Mathematics of Diffusion. 2nd Edition, Clarendon Press, Oxford.

[3] Churchill (1972) Operational Mathematics. 3rd Edition, McGraw-Hill, New York.

[4] Boyce, W.E. and Diprima, R.C. (1999) Equações Diferenciais Elementares e Problemas de Valores de Contorno. 6th Edition, LTC, Rio de Janeiro.

[5] Fox, R., Pritchard, P.J. and McDonald, A.T. (2011) Introdução à mecânica dos fluidos. 7th Edition, Tradução e revisão técnica de Ricardo Koury e Luiz Machado. Editora LTC, Rio de Janeiro.

[6] Araújo, J.C. and Márquez, R.G. (2018) Proposta alternativa para a estimativa de 
concentração de carbono em amostras metálicas de aço-carbono. Revista Eletrônica da Matemática, 4, 215-228.

[7] Callister, W.D. (2002) Ciência e Engenharia de Materiais: Uma Introdução. 5th Edition, LTC, Rio de Janeiro, Brazil.

[8] Bassanezi, R.C. and Ferreira, W.C. (1988) Equações Diferenciais com Aplicações. ed. Harbra, São Paulo, Brazil.

[9] Bassanezi, R.C. (2002) Ensino Aprendizagem com Modelagem Matemática. 3rd Edition, ed. Contexto, São Paulo, Brazil.

[10] Ruggiero, M.A.G. and Lopes, V.L.R. (2006) Cálculo Numérico Aspectos Teóricos e Computacionais. 2nd Edition, Pearson Makron Books, São Paulo, Brazil.

[11] Lima, E.L. (2016) Curso de Analise. Vol. 1, ed. IMPA, Rio de Janeiro, Brazil, 14.

[12] Neto, A.J.S. and Neto, F.D.M. (2005) Problemas Inversos. Conceitos Fundamentais e Aplicações. Ed. UERJ, Rio de Janeiro, Brazil.

[13] Bronson, R. and Costa, G. (2008) Equações Diferenciais. Bookman. 